\title{
Control Information Acquisition and Processing of the AMT System Based in LabVIEW and MATLAB
}

\author{
Zhisen Zhang*, Chengfu Yang** \\ *Sichuan University of Arts and Science \\ 519, Tashi Road, Dazhou City, \\ Sichuan,China \\ thrywoode163. com \\ **Sichuan University of Arts and Science \\ 519, Tashi Road, Dazhou City, \\ Sichuan,China \\ 443563624 @qq. com
}

\begin{abstract}
In this paper, the information acquisition and processing system is proposed for the automated mechanical transmission (AMT) control by LABVIEW and MATLAB. Firstly, an interface of the data acquisition was designed with the automated mechanical transmission system based in LabVIEW. Secondly, the data acquisition hardware was built by employing the single chip system incorporating with necessary sensors. Thirdly, the data acquired was transmitted into the data processing system built by MATLAB. By using the data processing system, the transmission status and driving parameters was presented in MATLAB which can be viewed easily and ready to be utilized further more.
\end{abstract}

Keywords: control information, acquisition, processing, AMT, LABVIEW, MATLAB

\section{Introduction}

The automated mechanical transmission (AMT) system is widely used in vehicles. Basically, the AMT system is composed of two parts, one is the mechanical transmission and the other is the shift mechanism which functions as an experienced personnel driver. By the shift mechanism, the mechanical transmission can be shifted from one gear to another according to the control strategies. For the purpose of obtaining efficient control strategies and implementing the control operation, the control information such as the driving motor (or engine) speed, clutch control data and mechanism motors driving currents data etc. have to be acquired and processed. In this paper, an information acquisition and processing system of the AMT based in LabVIEW and MATLAB was fabricated. By the LabVIEW, the acquired data can be presented in the Man-Machine interface and stored simultaneously. By MATLAB, the data acquired from different AMT locations which carries coupled information can be viewed easily and available for further utilized. 


\section{Overview of data acquisition and process techniques}

Data acquisition (DAQ) is the process in which the physical signals such as voltage, current, temperature, pressure and voice etc. can be measured, transmitted and stored in computer. Usually, a DAQ system is composed of sensors, DAQ measurement hardware and computer armed by programmable software. The schematic diagram of a typical DAQ system is shown in Figure 1. Compared with a traditional system, the computer DAQ system can provide more powerful, flexible and cheap resolutions by using standardized processing, displaying and communicating.

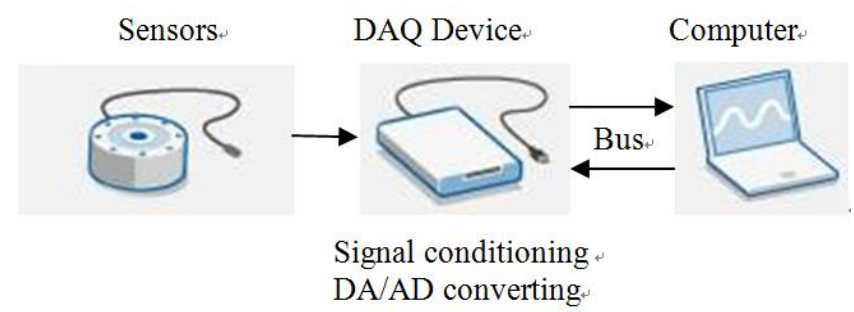

Fig. 1. Schematic diagram of DAQ system

A data processing system is a combination of machines, people, and processes that for a set of inputs produces a defined set of outputs. Data Processing ensures that the data is presented in a clean and systematic manner and is easy to understand and be used for further purposes. Usually, a data process involves steps like editing, coding, classification, tabulation and analysis which can be shown in figure 2 .

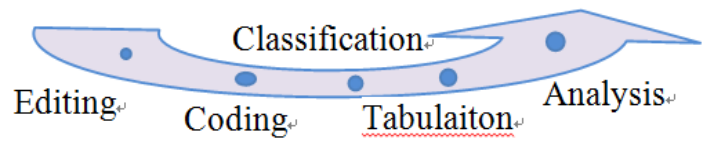

Fig. 2. Data processing steps

\section{Interface of the DAQ system}

In this research, the DAQ system includes sensors, data bus, and signal conditioning devices, AD/DA converters and man-machine interfaces. The transmission control unit (TCU) is powered by a single chip produced by Freescale (MC9S12DJ64). The shift mechanism has two driving motors. One of which selects the proper gear position and the other changes the gear by pushing the handle to the target position. During the changing gear process, the clutch must be interrupted for the protection of the transmission and shift mechanism. Thus the clutch openness was controlled as well. LabVIEW is systems engineering software for applications that require test, measurement, and control with rapid access to hardware and data insights. The gear control and clutch control interfaces are developed in the LabVIEW environment which are illustrated in figure 3 and figure 4. 


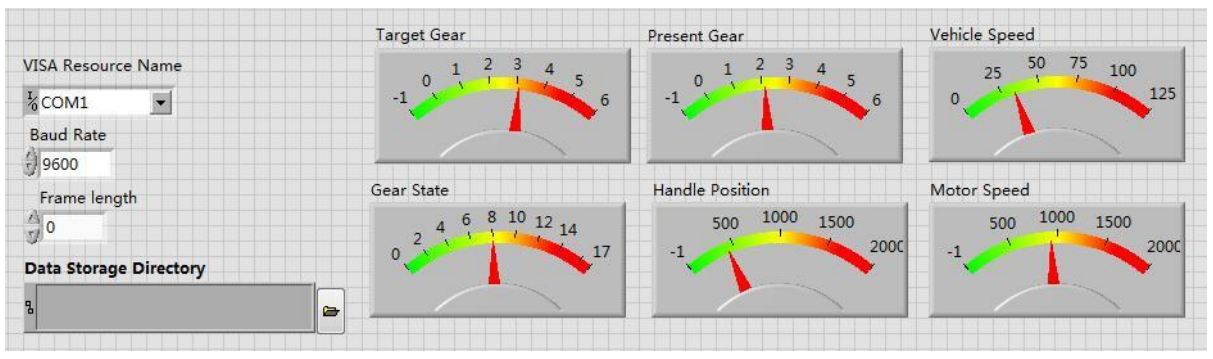

Fig. 3. Gear Control Interface

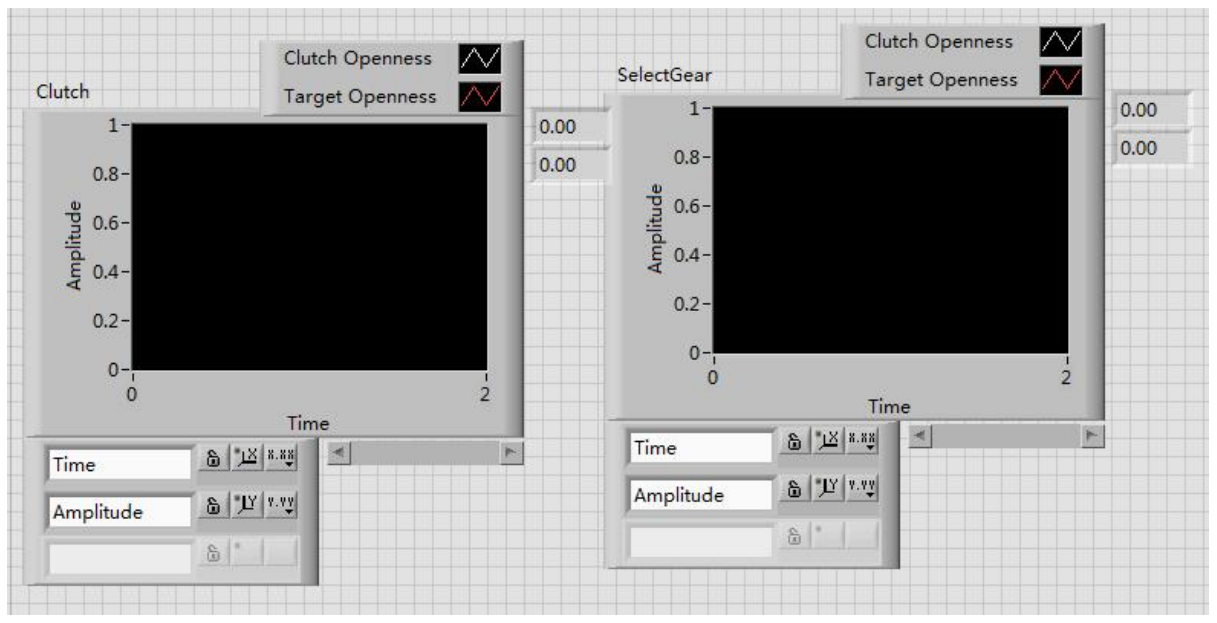

Fig. 4. Clutch Control Interface

\section{Data processing and results presentation}

MATLAB (matrix laboratory) is a multi-paradigm numerical computing environment developed by MathWorks. MATLAB allows matrix manipulations, plotting of functions and data, implementation of algorithms, creation of user interfaces, and interfacing with programs written in other software languages. The visualization of data in MATLAB environment by programming is user-friendly and can be completed easily. The acquired data such as gear status, engine speed, mechanism motors driving currents, handle position and transmission input/output speeds etc. are presented in figure 5 . 


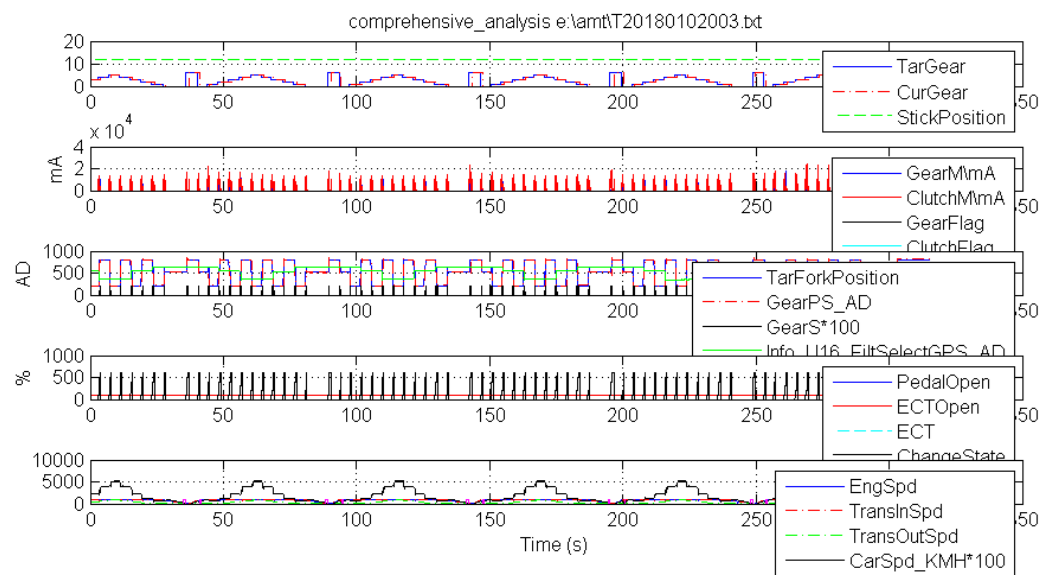

Fig. 5. Data processing by MATLAB programming

\section{Conclusions}

By using the data acquisition and processing system, the transmission status and driving parameters was presented in MATLAB which can be viewed easily and ready to be utilized further more in control strategy design and analysis. The method developed here can be used by analogous systems as well and is of practice.

\section{Acknowledgement}

This work was financially supported by the Education Department of Sichuan (No. 15ZA0318) and Sichuan University of Arts and Sciences.

\section{$7 \quad$ References}

1. XI Jun-qiang, WANG Leil, FU Wen-qin, LIANG Wan-wu, "Shifting Control Technology on Automated Mechanical Transmission of Pure Electric Buses", 2010, 30(1), pp.42-45, Transactions of Beijing Institute of Technology, 2010.

2. Y. Taguchi, et al., "Development of an Automated Manual Transmission System Based on Robust Design", SP-1760, pp.79-85, Transmission\& Driveline Systems Symposium, 2003.

3. Zhang Junzhi, Lu Qingchun, "Automated Mechanical Transmission in China", F2000A151, pp.1-7, Seoul 2000 FISITA World Automotive Congress, 2000.

4. Leping Yang, et al., "LabVIEW Programming and Application"(2nd edition, in Chinese), Publishing House of Electronic Industry, 2005.

5. National Instruments Corp, "LabVIEW Simulation Interface Toolkit User Guide". National Instruments, Inc. 2003.

6. V. Vitale, et al., "A Matlab based framework for the real-time environment at FTU", 2007,(82), pp.1089-1093, Fusion Engineering and Design,2007. 